\title{
Remoção biológica de nitrogênio em águas residuárias: uma revisão dos processos convencionais aos processos modernos
}

O lançamento de efluentes em corpos receptores ou em redes de esgoto deve ser monitorado e atender a legislação vigente. As máximas concentrações permitidas para o lançamento de nutrientes, especialmente o nitrogênio, se tornaram mais restritivas após 2005 . O nitrogênio é um nutriente que está presente em diferentes tipos de águas residuárias, como chorume, efluentes industriais e esgotamento sanitário. $O$ descarte deste tipo de efluente sem redução da carga de nitrogênio resulta em impactos sobre a fauna e flora de ecossistemas em geral. A remoção de nitrogênio está baseada na conversão de amônia a nitrito e nitrato (nitrificação) e posterior redução destes à nitrogênio gasoso (desnitrificação). Diversos métodos biológicos foram e têm sido desenvolvidos para remover nitrogênio de efluentes baseados em configurações complexas de reatores em série ou em sistemas unicompartimentados. Recentemente, novas rotas metabólicas envolvendo processos mais eficientes e econômicos têm sido apresentadas. Este trabalho apresenta uma revisão da literatura para os processos de nitrificação e desnitrificação convencional, nitrificação e desnitrificação simultânea, Sharon (Single Reactor System for High Activity Ammonia Removal Over Nitrite), Anammox (Anaerobic Ammonium Oxidation) e Canon (Completely Autotrophic Nitrogen Removal Over Nitrite). As principais características de cada processo foram abordadas, destacando-se as principais reações envolvidas, crescimento biológico, inibidores, requisitos e aplicações.

Palavras-chave: Remoção de Nitrogênio; Nitrificação; Desnitrificação; Sharon; Anammox; CANON.

\section{Biological nitrogen removal in wastewater: a review of conventional to the new conceptual processes}

\begin{abstract}
The discharge of effluents into water bodies or sewage networks must be monitored and comply the environmental laws. The maximum allowable concentrations for the release of nutrients, especially nitrogen, became more restrictive after 2005. Nitrogen is one of the nutrients that is present in several wastewater, such as leachate, industrial effluents and sewage. The disposal of this type of effluent without reducing in the nitrogen load results in impacts on the fauna and flora of ecosystems in general. The removal of nitrogen is based on the conversion of ammonia to nitrite and nitrate (nitrification) and subsequent reduction of these to nitrogen gas (denitrification). Several biological methods have been and are being developed to remove nitrogen from wastewater based on complex configurations of series reactors or unicompartmentalized systems. Recently, new metabolic routes involving more efficient and economical processes have been presented. This work presents a literature review for the process of conventional nitrification and denitrification, simultaneous nitrification and denitrification, Sharon (Single Reactor System for High Activity Ammonia Removal Over Nitrite), Anammox (Anaerobic Ammonium Oxidation) and Canon (Completely Autotrophic Nitrogen Removal Over Nitrite). The main characteristics of each process were discussed, such as biochemical reactions, biological growth, inhibitors, requirements and applications.
\end{abstract}

Keywords: Nitrogen Removal; Nitrification; Denitrification; Sharon; Anammox; CANON.

Topic: Engenharia Sanitária

Reviewed anonymously in the process of blind peer.
Received: $10 / 02 / 2018$

Approved: 24/03/2018
Camila Ferreira Alves (D)

Pontifícia Universidade Católica de Minas Gerais, Brasil

http://lattes.cnpq.br/3790882150777097

http://orcid.org/0000-0002-0330-8401

camila.ferreiraalves@hotmail.com

Carla Beatriz Casagrande Bortoluci (D)

Universidade Federal de Alfenas, Brasil

http://lattes.cnpq.br/0901953251853782

http://orcid.org/0000-0002-1060-4647

carlabeatriz.eng@outlook.com

Eduardo Paniguel Oliveira (id

Universidade Federal de Alfenas, Brasil

http://lattes.cnpq.br/5577552703010609

http://orcid.org/0000-0002-9492-5498

du.paniguel@gmail.com

DOI: 10.6008/CBPC2179-6858.2018.003.0015

\author{
Matheus Marques Pizzo (iD \\ Universidade Federal de Alfenas, Brasil \\ http://lattes.cnpq.br/0293536864887009 \\ http://orcid.org/0000-0003-3204-6129 \\ mat.pizzo@hotmail.com \\ Renata Piacentini Rodriguez (ic) \\ Universidade Federal de Alfenas, Brasil \\ http://lattes.cnpq.br/3494982072959140 \\ http://orcid.org/0000-0002-2837-3437 \\ piacentini.rodriguez@gmail.com
}

Referencing this:

ALVES, C. F.; BORTOLUCI, C. B. C.; OLIVEIRA, E. P.; PIZZO, M. M.; RODRIGUEZ, R. P.. Remoção biológica de nitrogênio em águas residuárias: uma revisão dos processos convencionais aos processos modernos. Revista Ibero Americana de Ciências Ambientais, v.9, n.3, p.174-187, 2018. DOI: http://doi.org/10.6008/CBPC2179$\underline{6858.2018 .003 .0015}$ 


\section{INTRODUÇÃO}

Os ciclos biogeoquímicos sofrem alterações devido às atividades humanas: queima de combustíveis fósseis, agricultura, produção de fertilizantes, entre outras ações. O desequilíbrio no ciclo do nitrogênio resulta no aumento das concentrações de óxido nitroso $\left(\mathrm{N}_{2} \mathrm{O}\right)$, gás de efeito estufa; acidificação de solos e ambientes aquáticos; perdas de nutrientes do solo, como o cálcio e o potássio; eutrofização, no qual há um aumento de algas e plantas aquáticas que causa depleção de oxigênio, provocando mortandade em peixes e prejuízo à fauna e a flora (CONLEY et al., 2009; SMITH et al.; 1999; VITOUSEK et al., 1997).

O nitrogênio pode ser apresentado sob formas: compostos orgânicos, amônia, nitrito, nitrato e nitrogênio gasoso. Em pH neutro, a amônia predomina na forma ionizada $\left(\mathrm{NH}_{4}^{+}\right)$, em pH básico, prevalece a forma livre $\left(\mathrm{NH}_{3}\right)$. Quanto maior o pH para uma mesma concentração de nitrogênio amoniacal, maior é a toxicidade (ZOPPAS et al., 2016). A presença de altas concentrações de nitrato ou nitrito podem causar risco a saúde humana, como a meta-hemoglobinemia (síndrome do bebê azul) (FAN et al., 1996).

Como a presença de nitrogênio na água pode causar problemas ambientais e para a saúde humana, tecnologias que englobam alta eficiência e baixo custo para tratamento de águas residuárias são essenciais. O processo mais comum e primeiramente estudado é a nitrificação e desnitrificação convencional. A nitrificação é definida pela oxidação da amônia a nitrito e posteriormente, nitrito a nitrato, na presença de oxigênio. A desnitrificação ocorre pela redução de nitrato a nitrogênio gasoso, em ambientes anaeróbios. Esse método apresenta alto custo devido a necessidade de aeração na etapa de nitrificação e adição de uma fonte externa de carbono na etapa de desnitrificação. Essas etapas são realizadas em reatores separados (AHN, 2006; CHARLEY et al., 1980; METCALF et al., 2003). A nitrificação e desnitrificação podem ocorrer simultaneamente em um único reator por meio do uso de biofilme, sendo sua realização possível em função do estabelecimento de gradiente de oxigênio na biomassa (MÜNCH et al., 1996).

Os processos mais novos têm como objetivo diminuir custos, aumentar a eficiência e praticidade. 0 processo Sharon tem o propósito de diminuir a demanda de oxigênio, selecionado a rota de nitrificação parcial, ou seja, oxidação da amônia nitrito, seguida de desnitrificação (HELLINGA et al., 1998). Anammox é o processo no qual a oxidação da amônia e redução do nitrito ocorrem simultaneamente em ambiente anóxico por um grupo de microrganismos específico, obtendo o nitrogênio gasoso como produto, sem requerimento algum de oxigênio e doador de elétrons (STROUS et al., 1998).

No processo Canon realizado-se em um sistema unicompartimenda a nitrificação parcial da amônia na presença de oxigênio, e posteriormente microrganismos Anammox realizam a oxidação da amônia residual na presença de nitrito, com significativa diminuição no consumo global de oxigênio (HU et al., 2014). O objetivo desta revisão é apresentar e discutir os processos de remoção de nitrogênio (Convencional, Sharon, Anammox e Canon), com destaque para as características de cada sistema e parâmetros operacionais, assim como as vantagens e desvantagens de cada processo. 


\section{DISCUSSÃO TEÓRICA}

\section{Nitrificação/Desnitrificação Convencional}

O processo convencional de remoção de nitrogênio utilizando microrganismos é conhecido como nitrificação e desnitrificação (KHIN et al., 2004). A nitrificação ocorre na presença de oxigênio e consiste na oxidação da amônia a nitrito e posteriormente, nitrito a nitrato (CHARLEY et al., 1980). Os microrganismos nitrificantes começaram a ser estudados em 1890 (WINOGRADSKY, 1890). A desnitrificação é realizada em ambientes anaeróbios, onde nitrato é reduzido a nitrogênio gasoso (NARKIS et al., 1979).

A nitrificação é catalisada pelas bactérias oxidadoras de amônia (BOAs) (Equação 1) e pelas bactérias oxidadoras de nitrito (BONs) (Equação 2). São microrganismos em sua maioria quimioautotróficos (DAIMS et al., 2015). O grupo de microrganismos mais conhecido responsáveis pela oxidação da amônia são as Nitrosomonas $\left(\mu_{\max }=0,77 \mathrm{~d}^{-1}\right)$, e da oxidação do nitrito são as Nitrobacter $\left(\mu_{\max }=1,08 \mathrm{~d}^{-1}\right)$. As Nitrosomonas possuem velocidade de crescimento menor do que as Nitrobacter, e por isso quase não há acumulo de nitrito (AHN, 2006; CHARLEY et al., 1980).

$$
\begin{array}{lrl}
\mathrm{NH}_{4}^{+}+1.5 \mathrm{O}_{2} \rightarrow \mathrm{NO}^{2-}+\mathrm{H}_{2} \mathrm{O}+2 \mathrm{H}^{+} & \Delta \mathrm{G}^{\mathrm{o \prime}}=-275 \mathrm{~kJ} \mathrm{~mol}^{-1} \\
\mathrm{NO}^{-2}+0,5 \mathrm{O}_{2} \rightarrow \mathrm{NO}^{-3} & \Delta \mathrm{G}^{\mathrm{o \prime}}=-74 \mathrm{KJ} \cdot \mathrm{mol}^{-1}
\end{array}
$$

Segundo as equações 1 e 2 , são necessários 4,57mg de oxigênio para oxidar a amônia a nitrato, sendo $3,43 \mathrm{mg}$ de $\mathrm{O}_{2}$ para oxidar $1 \mathrm{mg}$ de $\mathrm{NH}^{4+}$ e $1,14 \mathrm{mg}$ de $\mathrm{O}_{2}$ para oxidar $1 \mathrm{mg}$ de $\mathrm{NO}^{-2}$. A redução de nitrato a nitrito, óxido nítrico (NO), óxido nitroso $\left(\mathrm{N}_{2} \mathrm{O}\right)$ e nitrogênio gasoso $\left(\mathrm{N}_{2}\right)$ é conhecida como desnitrificação, podendo ser observada na Equação 3 (METCALF et al., 2003):

$\mathrm{NO}_{3}^{-} \rightarrow \mathrm{NO}_{2} \rightarrow \mathrm{NO} \rightarrow \mathrm{N}_{2} \mathrm{O} \rightarrow \mathrm{N}_{2}$

A desnitrificação pode ser realizada por microrganismos heterotróficos e autotróficos. A desnitrificação heterotrófica é a mais empregada, definida por utilizar a matéria orgânica como fonte de carbono e de energia. As bactérias heterotróficas apresentam alta velocidade de crescimento específico $\left(\mu_{\max }=7,2 \mathrm{~d}^{-1}\right)$, valor muito maior quando comparado com as bactérias nitrificantes. Oxigênio dissolvido deve ser mantido em baixas concentrações para não interferir na desnitrificação (METCALF et al, 2003).

A fonte de carbono orgânica é utilizada como doadores de elétrons, podendo ser originada da própria água residuária ou inserida externamente, tendo como exemplos o metanol, acetato, etanol, lactato e glicose (AHN, 2006; AKUNNA et al., 1993; NARKIS et al., 1979). A Equação 4 mostra a desnitrificação utilizando como fonte de carbono o metanol e o nitrato como aceptor de elétrons. São consumidos 2,47g de metanol para a redução de $1 \mathrm{~g}$ de nitrato (AHN, 2006):

$\mathrm{NO}_{3}^{-}+1,08 \mathrm{CH}_{3} \mathrm{OH}+0,24 \mathrm{H}_{2} \mathrm{C} \rightarrow 0,056 \mathrm{C}_{5} \mathrm{H}_{7} \mathrm{O}_{2} \mathrm{~N}+0,47 \mathrm{~N}_{2}+1,68 \mathrm{H}_{2} \mathrm{O}+\mathrm{HCO}_{3}^{-}$

A temperatura, $\mathrm{pH}$, concentração de oxigênio dissolvido (OD), presença de inibidores e alcalinidade interferem diretamente na eficiência da nitrificação e desnitrificação. Na Tabela 1 estão representadas as 
principais faixas de operação desses parâmetros. Os metais pesados podem causar toxicidade aos microrganismos e diminuir a eficiência de remoção de nitrogênio. Sun et al. (2016) identificaram uma nova bactéria nitrificante heterotrófica e resistente a metais, nomeada como Cupriavidus sp. S1, e concluíram que o nitrogênio pode ser removido mesmo com altas concentrações de metal pesado envolvido.

Tabela 1: Faixa de Operação para Nitrificação e Desnitrificação.

\begin{tabular}{|c|c|c|}
\hline Parâmetro & Nitrificação & Desnitrificação \\
\hline $\begin{array}{lr}\text { Gêneros } & \text { de } \\
\text { microrganismos } & \text { que } \\
\text { atuam no processo } & \\
\end{array}$ & $\begin{array}{l}\text { Nitrosomonas, Nitrobacter, Nitrosococcus, } \\
\text { Nitrosospira, Nitrosolobus, Nitrococcus, } \\
\text { Nitrospira, Nitrospina, entre outas }{ }^{\mathrm{a}}\end{array}$ & $\begin{array}{l}\text { Archromobacter, Acinetobacter, Alcaligenes, } \\
\text { Bacillus, Chromobacterium, Flavobacterium, } \\
\text { Paracoccus, Pseudomonas, entre outras }{ }^{\mathrm{a}}\end{array}$ \\
\hline Faixa de temperatura (oC) & $25-36^{\text {bce }}$ & $10-30^{\text {ce }}$ \\
\hline $\mathrm{pH}$ & $7-8^{a}$ & $6,5-8^{\text {abe }}$ \\
\hline $\begin{array}{l}\text { Oxigênio dissolvido (mg.t- } \\
\left.{ }^{-}\right)\end{array}$ & $2-3^{\text {de }}$ & Inferior a $1^{\mathrm{e}}$ \\
\hline Inibidores & Alta concentração de amônia e ácido nitroso ${ }^{b}$ & Alta concentração de oxigênio dissolvidoe \\
\hline Alcalinidade $\left(\mathrm{mgCaCO}_{3}\right)$ & Consome alcalinidade: $7,14^{\text {ae }}$ & Gera alcalinidade: $3,57^{a}$ \\
\hline
\end{tabular}

Fonte: a Metcalf et al. (2003), b Yoo et al. (1999), c Obaja et al. (2003), d Daniel et al. (2009), e Surampalli et al. (1997).

O processo de remoção de nitrogênio pelo método convencional geralmente ocorre em duas unidades separadas, em uma ocorre a oxidação da amônia e nitrito e na outra, a redução do nitrato a nitrogênio gasoso. Quando utilizado a desnitrificação seguida da nitrificação, uma parte do efluente nitrificado é recirculado para o primeiro reator, e apresenta as vantagens de utilizar a matéria orgânica como doador de elétrons na desnitrificação e evitar a toxicidade de compostos orgânicos aos microrganismos nitrificantes, pois estes são removidos no primeiro estágio (KOREN et al., 2000).

A nitrificação e desnitrificação simultânea (NDS) são realizadas em um único reator em condições iguais. Esse tipo de sistema apresenta vantagem sobre os tratamentos convencionais, visto que resulta na economia de implantação do sistema e espaço. A NDS ocorre por causa de dois fenômenos: físico e biológico. O fenômeno físico acontece porque existe um gradiente de concentração de oxigênio no floco microbiano ou biofilme. A diferença de concentração de oxigênio na biomassa permite que ocorra nitrificação e desnitrificação simultaneamente (MÜNCH et al., 1996), conforme representado pela Figura 1.

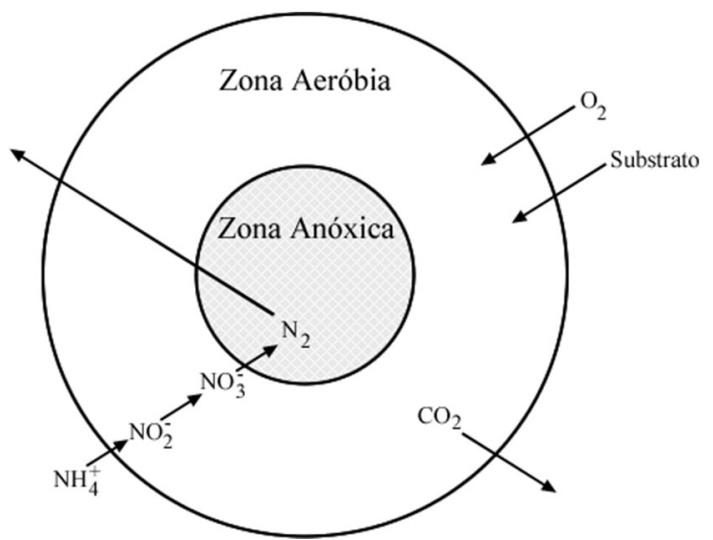

Figura 1: Floco biológico representando a NDS. Fonte: Adaptado de Metcalf et al. (2003).

A explicação biológica está em contraste com o princípio de nitrificação e desnitrificação, devido a constatações de bactérias desnitrificantes aeróbias e nitrificantes heterotróficas (ARTS et al., 1995; MÜNCH et al., 1996; ROBERTSON et al., 1984). Li et al. (2015) avaliaram a remoção de nitrogênio por nitrificação 
heterotrófica e desnitrificação aeróbia de uma bactéria acumuladora de fosfato denominada Pseudomonas stutzeri YG-24 e concluíram que essa cepa é um candidato adequado para remover simultaneamente o nitrogênio e o fosfato no tratamento de águas residuárias.

O controle operacional e ambiental é uma ferramenta que pode ser utilizada para favorecer a NDS. Os principais fatores para desenvolvimento e estabilização do processo são: baixas concentrações oxigênio dissolvido; elevado tempo de retenção de sólidos (TRS); quantidade suficiente de matéria orgânica disponível; temperatura que favoreça a nitrificação (BUENO, 2011).

Timberlake et al. (1988) realizaram o tratamento de esgoto em um reator de biofilme, utilizando a nitrificação e desnitrificação simultânea, no qual oxigênio foi inserido no centro do meio suporte permeável. Os autores concluíram que este sistema apresenta algumas vantagens: isolamento das bactérias nitrificantes em uma zona de alta concentração de amônia e oxigênio dissolvido para maximizar a conversão de amônia em nitrato; isolamento das bactérias desnitrificantes, onde há o contato com alta carga orgânica, aumentando assim, a eficiência de remoção de nitrato; camada anaeróbia perto da fonte de carbono para aumentar a transferência de massa e minimizar a produção de sólidos.

A nitrificação e desnitricação simultânea também são possíveis de serem realizadas por células de combustíveis microbianas (PARK et al., 2017; RYU et al., 2013; VIRDIS et al., 2010). Esse processo apresenta vantagens, como maior eficiência na remoção de contaminantes, geração de bioeletricidade, e não há necessidade de adição de fonte de carbono, além de gerar menos lodo, por esses motivos atualmente está sendo estudado como alternativa ao processo convencional (SUN et al., 2016).

O processo de nitrificação e desnitrificação convencional apresenta alto custo devido à necessidade de adicionar uma fonte de carbono orgânica na etapa de desnitrificação e aeração na etapa de nitrificação. Com o intuito de diminuir custos e aumentar a eficiência de remoção de nitrogênio em tratamento de águas residuais, foram criados outros sistemas e metodologias: Anammox, Sharon e Canon.

\section{SHARON}

O processo SHARON (Single Reactor High Activity Ammonia Removal Over Nitrite) é uma técnica empregada para o tratamento biológico de efluentes com altas cargas de nitrogênio, proposto na Universidade Técnica de Delft, na Holanda (HELLINGA et al., 1998) e baseia-se numa interrupção no processo de nitrificação. A nitrificação é bloqueada em uma etapa intermediária, mantendo o nitrogênio na forma de nitrito para que, de maneira anaeróbia, haja uma futura conversão de nitrito a nitrogênio gasoso (desnitrificação heterotrófica pela adição de uma fonte externa de carbono), economizando energia e doadores de elétrons (DERKS, 2007). Isso por que, em altas temperaturas (acima de $28^{\circ} \mathrm{C}$ ), as Nitrobacter (oxidadoras de nitrito) possuem uma velocidade de crescimento menor do que as Nitrosomonas (oxidadoras de amônia) (VAN DONGEN et al., 2001).

Além disso, devido ao curto TDH (aproximadamente 1 dia), as bactérias nitrito-oxidantes (Nitrobacter) são lavadas do reator. Assim, o descarte dos organismos que oxidam nitrito é feito de acordo com a idade do lodo, que deve ser escolhida baseada na idade mínima para manutenção dos organismos que 
oxidam amônia (VERSTRAETE et al., 1998). A temperatura, em conjunto ao curto TDH, torna-se um fator de seletividade. A $35^{\circ} \mathrm{C}$, a máxima velocidade de crescimento $\left(\mu_{\text {máx }}\right)$ de bactérias nitrito-oxidantes é aproximadamente a metade do que a das amônio-oxidantes $\left(0,5\right.$ e $1 \mathrm{dia}^{-1}$, respectivamente) (VERSTRAETE et al., 1998; JETTEN et al., 2001; VAN KEMPEN et al., 2001).

Os parâmetros operacionais mais importantes do processo Sharon são: pH, temperatura, tempo de retenção hidráulica, tempo de retenção de sólidos, presença de bicarbonato e oxigênio dissolvido (OD). A alta taxa de crescimento específico bacteriana é favorecida com uma temperatura de $30^{\circ} \mathrm{C} \mathrm{em}$ uma faixa de pH entre 8 e 8,5. Utilizando esses parâmetros é possível diminuir custos e demanda de espaço, visto que não é necessário retenção de lodo. A constante de saturação de oxigênio (ks) das BOAs $\left(0,3 \mathrm{mg} \cdot \mathrm{L}^{-1}\right)$ é menor que a das BONs (1,1 mg. $\left.\mathrm{L}^{-1}\right)$, isso indica que as BOAs possuem vantagem em ambientes com limitação de oxigênio. O processo de conversão de amônia a nitrito é um processo acidificante, e pode ser neutralizado através do bicarbonato encontrado no efluente rico em amônio. No processo de nitrificação, a oxidação do amônio a nitrito necessita de 2 mols de bicarbonato para cada mol de amônio (SRI SHALINI et al., 2012).

$$
\begin{array}{lr}
\mathrm{NH}_{3}+1,5 \mathrm{O}_{2} \rightarrow \mathrm{NO}_{2}{ }^{-}+\mathrm{H}_{2} \mathrm{O}+\mathrm{H}^{+} & \Delta G^{\circ}=-384,40 \mathrm{~kJ} \\
\mathrm{NH}_{3}+2 \mathrm{O}_{2} \rightarrow \mathrm{NO}_{3}{ }^{-}+\mathrm{H}_{2} \mathrm{O}+\mathrm{H}^{+} & \Delta G^{\circ}=-267,84 \mathrm{~kJ}
\end{array}
$$

Silva Filho (2009) descreve, por meio de equações, as vantagens energéticas da nitrificação parcial com desnitrificação via nitrito. Na Equação 5 é retratada a reação via nitrificação parcial, ao passo que a Equação 6 se relaciona com a nitrificação convencional. A Equação 7 destaca o processo de desnitrificação via nitrito. Já a Equação 8 refere-se à desnitrificação convencional. Com isto, o processo garante uma economia de $25 \%$ de oxigênio e $40 \%$ de carbono orgânico (VAN DONGEN et al., 2001).

$$
\begin{array}{ll}
8 \mathrm{NO}_{2}{ }^{-}+3 \mathrm{CH}_{3} \mathrm{COO}^{-}+5 \mathrm{H}^{+} \rightarrow 4 \mathrm{~N}_{2}+6 \mathrm{HCO}_{3}{ }^{-}+4 \mathrm{H}_{2} \mathrm{O} & \Delta G^{\circ}=-3007 \mathrm{~kJ} \\
8 \mathrm{NO}_{2}{ }^{-}+5 \mathrm{CH}_{3} \mathrm{COO}^{-}+3 \mathrm{H}^{+} \rightarrow 4 \mathrm{~N}_{2}+10 \mathrm{HCO}_{3}{ }^{-}+4 \mathrm{H}_{2} \mathrm{O} & \Delta G^{\circ}=-4076 \mathrm{~kJ}
\end{array}
$$

Mulder et al. (1997) frisam que os pontos positivos do processo SHARON são os baixos custos de implantação, operação e manutenção, visto que o sistema é constituído por apenas um reator e o custo com aeração é diminuído. Apesar disto, a manutenção da temperatura no sistema é a principal dificuldade. Hellinga et. al. (2001) descreveram o primeiro processo em larga escala para tratamento de efluentes, com um reator de $1800 \mathrm{~m}^{3}$. O procedimento obteve sucesso, com uma conversão de $90 \%$ de amônia. O processo apresentou uma boa estabilidade, sendo facilmente controlado pelos períodos de aeração.

Recentemente, alguns pesquisadores concentraram a aplicação do processo SHARON para o tratamento de lixiviados. Um estudo realizado por Liang et al. (2007) em um reator FBBR mostrou um lixiviado com carga de amônia de $0,2-1,0 \mathrm{~kg}$ de $\mathrm{NH}_{4}{ }^{+}-\mathrm{N} \cdot \mathrm{m}^{-3} \cdot \mathrm{d}^{-1}$, e alcançou $94 \%$ de nitrificação parcial com uma relação de $\mathrm{NH}_{4}-\mathrm{N} / \mathrm{NO}_{2}-\mathrm{N}$ 1,0-1,4. Spagni et al. $(2008 ; 2009)$ estudaram a remoção de nitrogênio via nitrito em um Reator de batelada sequencial (SBR) contendo lixiviação de aterro sanitário. A taxa de nitrificação e remoção de nitrogênio foi superior a 98\% e 95\%, respectivamente. 
O processo SHARON para tratamento de lixiviados também foi demonstrado por Wei et al. (2010), com a remoção simultânea de conteúdo orgânico e nitrogênio do lixiviado pelo sistema UASB-SBR. As concentrações influentes de NH4-N e DQO foram 2012 e $6537 \mathrm{mg} \cdot \mathrm{L}^{-1}$ atingindo apenas 5 e $354 \mathrm{mg} \cdot \mathrm{L}^{-1}$, respectivamente. As eficiências de remoção foram de $99,7 \%$ e $94,5 \%$. Todos os estudos acima demonstraram que o processo SHARON é viável para remoção de amônia e pode ser usado como pré-tratamento para o processo Anammox.

\section{ANAMMOX}

Uma rota alternativa para a oxidação da amônia ocorre no processo Anammox (Anaerobic Ammonium Oxidation). Neste processo, a oxidação da amônia ocorre de forma concomitante à redução do nitrito em meio anaeróbio, obtendo como principal produto o gás nitrogênio (Equação 9) (STROUS et al., 1998). Este processo é realizado por bactérias e tem diversas aplicações, como o tratamento de esgoto sanitário, efluentes industriais, digestores, tratamento de chorume (ZHANG et al., 2014), efluente de suinocultura (CHIUMENTI, 2015; SUTO et al., 2017) e tratamento de águas salinas (RIOS-DEL TORO et al., 2017).

$1 \mathrm{NH}_{4}^{+}+1,32 \mathrm{NO}_{2}^{-}+0,066 \mathrm{HCO}_{3}^{-}+0,13 \mathrm{H}^{+} \rightarrow 1,02 \mathrm{~N}_{2}+0,26 \mathrm{NO}_{3}^{-}+0,066 \mathrm{CH}_{2} \mathrm{O}_{0,5} \mathrm{~N}_{0,15}+2,03 \mathrm{H}_{2} \mathrm{O}$

O processo Anammox é novo comparado a outras formas de remoção de nitrogênio. A descoberta desta suposta atividade bacteriana foi primeiramente publicada por Van de Graaf et al. (1995). Somente em 1999, a bactéria foi isolada e devidamente identificada (STROUS et al., 1999). Após estes estudos, houve um aumento significativo no número de artigos publicados com o tema Anammox, partindo de 1 a 5 artigos por ano entre 1995 e 2000, chegando até 191 artigos publicados somente em 2012 (ZHANG et al., 2014). Atualmente são conhecidos 5 gêneros de bactérias que realizam o processo Anammox: Candidatus Brocadia, Candidatus Kuenenia, Candidatus Jettenia, Candidatus Scalindua e Candidatus Anammoxoglobus (KUENEN, 2008).

As principais vantagens deste processo é a robustez, não necessidade de adição de fontes de carbono e menor consumo de oxigênio, comparado aos demais processos de remoção de nitrogênio (a demanda de oxigênio somente é necessária quando não há uma fonte de nitrito) (MA et al., 2016). Outra vantagem deste processo é a sua versatilidade, podendo ser utilizado para variadas aplicações de nitrogênio. Na literatura recente são encontrados valores que variam de $70 \mathrm{mg} \cdot \mathrm{L}^{-1}$ ou $0.09 \mathrm{kgN} \cdot \mathrm{m}^{-3} \cdot \mathrm{d}^{-1}$ (REINO et al., 2017) a 1250mg. $\mathrm{L}^{-}$ ${ }^{1}$ e $10 \mathrm{kgN} \cdot \mathrm{m}^{-3} \cdot \mathrm{d}^{-1}$ (QIN et al., 2017). Porém, ainda há casos encontrados em literatura de aplicação de nitrogênio total de $3000 \mathrm{mg} \cdot \mathrm{L}^{-1}\left(1,65 \mathrm{kgN} \cdot \mathrm{m}^{-3} \cdot \mathrm{d}^{-1}\right)$ em tratamento de chorume em escala laboratorial (MIAO et al., 2016) e, em outro caso obteve-se eficiência de $70 \%$ de remoção com aplicação de nitrogênio com concentração de $660 \mathrm{mg} \cdot \mathrm{L}^{-1}$ e carga de $89,1 \mathrm{kgN} \cdot \mathrm{m}^{-3} \cdot \mathrm{d}^{-1}$, operando com TDH de 0,21 horas (TANG et al., 2011).

A principal desvantagem deste reator é o baixo crescimento celular, demandando longo tempo de partida. Valores médios da taxa de crescimento bacteriano máximo $\left(\mu_{\max }\right)$ são encontrados na literatura na faixa de 0,05 a $0,23 \mathrm{~d}^{-1}$, porém dois autores obtiveram valores de $0,33 \mathrm{~d}^{-1}$ para o gênero Brocadia sp. por 
meio de adaptação com redução progressiva do tempo de detenção celular (LOTTI et al., 2015; ZHANG et al., 2017).

Devido ao baixo crescimento celular, a maioria dos reatores Anammox possuem algum mecanismo para contenção celular. Os mecanismos mais comuns são a formação de biofilme em estruturas fixas ou móveis, como espumas de poliéster, ou pode ser feita através da formação de grânulos ou então utilizado reatores de mistura em batelada. É também presente na literatura a utilização de dois reatores como o caso de Sharon-Anammox de forma mais intensa até os anos de 2006. Recentemente (2006 a 2012), houve o aumento de publicações utilizando reatores únicos, nomeados como Canon, Deammonification, NDS, Oland e Sharon-Anammox, apresentados em ordem decrescente (ZHANG et al., 2014).

A atividade anammox é influenciada principalmente pelos parâmetros como temperatura, pH, OD, DQO e carga de nitrogênio. Na natureza, é observado o processo Anammox ocorrendo em faixas de temperatura de -4 até $80^{\circ} \mathrm{C}$, porém a maioria dos estudos em reatores são realizados entre 10 e $45 \circ \mathrm{C}$. A faixa ótima de temperatura presente na literatura encontra-se entre 25 e $40 \circ \mathrm{C}$, apresentando algumas diferenças para cada um dos 5 gêneros (TOMASZEWSKI et al., 2017). No geral, as bactérias Anammox demonstram redução drástica da atividade em baixas temperaturas, mas apresentaram capacidade de recuperação após choques de temperaturas menores que 11으 (LAURENI et al., 2016).

$\mathrm{O}$ pH além influenciar a atividade bacteriana está relacionado com a concentração de compostos tóxicos como a amônia livre $\left(\mathrm{NH}_{3}\right)$ e o ácido nitroso $\left(\mathrm{HNO}_{2}\right)$ que fortemente inibem o processo Anammox. $\mathrm{A}$ faixa do $\mathrm{pH}$ para o crescimento dos 5 gêneros bacterianos é entre 6,5 e 8,5, usualmente na literatura encontra-se valores levemente básicos de 7,2 a 8, sendo que o ponto ótimo é próximo a 8 (TOMASZEWSKI et al., 2017).

A concentração de oxigênio dissolvido no meio líquido é fundamental para a seleção das bactérias que realizam Anammox, pois em concentrações acima de $2 \mathrm{mg} / \mathrm{L}$ há o favorecimento do crescimento das Nitrossomonas $s p$. que utilizam do oxigênio para oxidar a amônia e inibição das bactérias Anammox (KUENEN, 2008). Porém, um estudo realizado em reator do tipo Canon (nitrificação parcial/Anammox), demonstrou-se que existe uma relação positiva entre OD e carga de nitrogênio. Em tal estudo, obteve-se eficiências acima de $90 \%$ de remoção de Nitrogênio mesmo com OD elevado $\left(2,3\right.$ a 2,4 mg. $\left.\mathrm{L}^{-1}\right)$ para altas concentrações de nitrogênio $2,2 \mathrm{~g} \cdot \mathrm{L}^{-1}$, demonstrando que o interior do biofilme proporcionava um ambiente anóxico para a atividade anammox (WEN et al., 2017). O processo Anammox, apesar de recente, tem sido alvo de pesquisas para consolidação da técnica e mostra-se economicamente favorável devido a possibilidade de atuar em reatores únicos, com menor ou nenhuma demanda de oxigênio, obtendo elevada eficiência na remoção de nitrogênio.

\section{CANON}

O processo CANON (Completely Autotrophic Nitrogen removal Over Nitrite) é um processo de remoção nitrogênio de forma autotrófica onde são consumidos $63 \%$ menos de oxigênio e $100 \%$ menos de carbono orgânico comparado ao processo convencional de remoção de nitrogênio de águas residuárias (KUAI 
et al., 1998; KHIN et al., 2004). O sistema CANON difere do processo de remoção de nitrogênio de forma convencional devido a capacidade de receber elevadas cargas de nitrogênio e principalmente por não requerer matéria orgânica (KHAJAH et al., 2016).

A proposição do modelo CANON resultou da observação de uma grande quantidade de remoção de nitrogênio (90\%) como nitrogênio elementar a partir de águas residuais altamente carregadas com amônia e com baixas concentrações de carbono orgânico (HIPPEN et al., 1997; HELMER et al., 1999; KUAl et al., 1998; SIEGRIST et al., 1998; HELMER et al., 2001; KOCH et al., 2000). A partir dessas observações foi desenvolvido um novo processo patenteado por Dijkman et al. (1999) que combina dois processos biológicos para remoção de nitrogênio por organismos completamente autotrófico que se desenvolvem em grânulos em apenas um reator.

A remoção de nitrogênio pelo CANON ocorre em duas etapas sequenciais: a primeira etapa é a nitrificação parcial e a segunda etapa é oxidação da amônia por organismos anaeróbios, podendo ser executado em um mesmo reator aerado (BAGCHI et al., 2010; HU et al., 2014). Essas etapas podem acontecer devido à interação estável entre duas populações bacterianas, as bactérias aeróbias do tipo Nitrosomonas e as bactérias anaeróbias do tipo Planctomicetos (Anammox) (LIANG et al., 2014; SLIKERS et al., 2003).

O processo CANON depende de parâmetros operacionais específicos como oxigênio dissolvido, carga de amônia, espessura do biofilme, temperatura e a concentração de nitrito devem ser bem ajustados para coexistirem as oxidantes anaeróbias e aeróbias de amônia. A maioria dos sistemas CANON relatados na literatura foi operado a temperaturas variando entre 30 a $35^{\circ} \mathrm{C}$ com uma máxima taxa de remoção de nitrogênio de 0,075 a $1,5 \mathrm{kgNm}^{-3} \mathrm{~d}^{1}$ (SLIKERS et al., 2003).

Sob a condição de limitação de oxigênio e de baixa quantidade de receptores de elétron, a amônia é nitrificada parcialmente a nitrito por nitrificadores aeróbios (Nitrosomonas) (HANAKI et al., 1990) (Equação 10), subsequentemente as bactérias anaeróbias Anammox oxidantes de amônia (Planctomycete) consomem o nitrito gerado da etapa anterior convertendo a amônia em gás nitrogênio (Equação 11). A combinação da equação I com a equação II resulta na remoção completa de nitrogênio (Equação 12).

$$
\begin{aligned}
& \mathrm{NH}_{4}^{+}+1,5 \mathrm{O}_{2} \rightarrow \mathrm{NO}_{2}^{-}+2 \mathrm{H}^{+} \mathrm{H}_{2} \mathrm{O} \\
& \mathrm{NH}_{4}^{+}+1,3 \mathrm{NO}_{2}^{-} \rightarrow 1,02 \mathrm{~N}_{2}+0,26 \mathrm{NO}_{3}^{-}+2 \mathrm{H}_{2} \mathrm{O} \\
& \mathrm{NH}_{4}^{+}+0,85 \mathrm{O}_{2} \rightarrow 0,435 \mathrm{~N}_{2}+0,13 \mathrm{NO}_{3}^{-}+1,4 \mathrm{H}^{+}
\end{aligned}
$$

O processo CANON requer aeração limitada e essa situação é favorecida utilizando-se reatores com fluxo ascendente (HU et al., 2014). Em situações onde há limitação de oxigênio, o processo de nitrificação completa é desfavorecido, ocorrendo apenas a conversão parcial da amônia para nitrito presente limitandose a conversão de nitrito para nitrato. Uma concentração de oxigênio dissolvido de até $0,5 \mathrm{mg} \cdot \mathrm{L}^{-1}$ não tem efeito sobre a oxidação da amônia, mas a oxidação de nitrito é fortemente inibida em reatores com crescimento em suspensão (HANAKI et al., 1990). Nas condições de oxigênio limitado, as bactérias oxidantes 
de nitrito têm que competir pelo oxigênio com as oxidantes de amônia aeróbia e com nitrito com as bactérias oxidantes de amônia anaeróbia (ABELING et al., 1992).

O convívio das bactérias anaeróbias e aeróbias sob a condição de oxigênio limitado resulta em uma conversão da amônia quase completa em gás nitrogênio e pequenas quantidades de nitrato. A amônia pode inibir o processo caso a produção de nitrito seja inferior a concentração de amônia, ocasionando uma baixa remoção de nitrogênio. As bactérias aeróbias e as bactérias anaeróbias oxidadoras de amônia podem conviver de forma simbiótica devido a formação dos grânulos. O nitrito produzido nas camadas externas é utilizado pelos organismos Anammox que são ativos nas camadas internas (VAN HULLE et al., 2010).

Em um estudo realizado por um período de 508 dias em um biorreator de fluxo ascendente em condições anóxicas para tratar águas residuais industriais com alta concentração de nitrogênio e amônia foi possível obter informações referentes a eficiência do sistema CANON. O estudo foi divido em três fases com concentrações de hidróxido de tetrametilamônio variando de 200, 500 e $1000 \mathrm{mg}^{-1} \mathrm{~L}^{-1}$ respectivamente, com a adição do hidróxido de tetrametilamônio marcado com ${ }^{15} \mathrm{~N}$ como substrato no biorreator e pH mantido entre 7 e 8 . Observou-se que o hidróxido de tetrametilamônio foi quase completamente biodegradável no sistema CANON após um longo período de adaptação do biorreator (300 dias). A eficiência média de remoção de nitrogênio foi superior a 90\%. A trimetilamina e a metilamina, segundo o estudo, foram os principais compostos intermediários de biodegradação do hidróxido de tetrametilamônio. Pode-se observar que a partir do hidróxido de tetrametilamônio marcado com ${ }^{15} \mathrm{~N}$ o sistema CANON foi satisfatório na conversão do mesmo em gás nitrogênio (CHEN et al., 2016).

No sistema CANON, a taxa de crescimento de bactérias Anammox é extremamente baixa, assim é necessário um sistema eficiente para a retenção do lodo e um longo período de partida para obter resultados satisfatórios de remoção de nitrogênio na água a ser tratada (STROUS et al., 1998; JETTEN et al., 1998). Em outro experimento utilizou-se um reator de fluxo descendente tipo wetland e aeração intermitente. A recirculação intermitente foi proposta para criar condições necessárias para ocorrer o processo CANON, basicamente, limitar a disponibilidade de oxigênio. A água residual utilizada foi sintética visando simular as águas residuais domésticas após o tratamento secundário (altas concentrações de carbono inorgânico (150 mg.L-1), nitrogênio total (16, $\pm 0,29 \mathrm{mg} . \mathrm{L}-1)$, amônia $(14,63 \pm 0,29 \mathrm{mg} . \mathrm{L}-1)$ e DQO $(102 \pm 1,61 \mathrm{mg} . \mathrm{L}-1))$. As bactérias oxidantes de amônia e as bactérias Anammox apresentaram aumento de atividade metabólica em altas concentrações de carbono inorgânico, enquanto para as para bactérias oxidantes de nitrito não se observou efeito algum. O tratamento CANON resultou em remoções de $67 \%$ de nitrogênio total

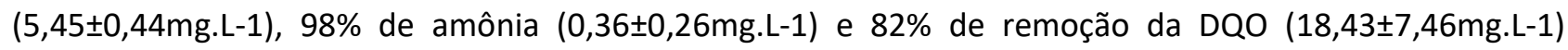
(KHAJAH et al., 2016).

\section{CONCLUSÕES}

Os processos mais recentes de remoção de nitrogênio trazem vantagens consideráveis quando comparados ao processo convencional (nitrificação autotrófica completa seguida de desnitrificação heterotrófica). Temperatura, pH, concentração de oxigênio dissolvido, presença de inibidores, alcalinidade e 
a presença de metais pesados são parâmetros que interferem diretamente na eficiência do processo convencional. Para o processo convencional, utilizam-se dois reatores e enquanto o processo de nitrificação e desnitrificação simultânea pode ocorrer em um único reator a partir da ocorrência de um gradiente de concentração de oxigênio no biofilme ou floco microbiano e do sintrofismo entre bactérias desnitrificantes aeróbias e nitrificantes heterotróficas. O processo SHARON baseado na nitrificação parcial requer baixo TDH e as altas temperaturas para promover a seletividade de microrganismos nitritantes no reator.

A eficiência do sistema depende do $\mathrm{pH}$, temperatura, velocidade específica do crescimento bacteriano, vazão específica de alimentação e oxigênio dissolvido, e tem como principal vantagem a economia de implantação, operação e manutenção. Os sistemas do tipo ANAMMOX também requerem alto controle operacional, entretanto, dispensam o fornecimento de oxigênio e de doador de elétrons, tem como vantagem a robustez e a capacidade de acomodar elevadas cargas de nitrogênio. São os sistemas mais compactos e econômicos, porém, demandam alto tempo de partida quando não se possui inóculo específico, possui baixo crescimento celular e dependem de mecanismos para retenção celular.

O processo é influenciado pela temperatura, $\mathrm{pH}$, oxigênio dissolvido, demanda química de oxigênio e carga de nitrogênio. O processo CANON tem como premissa reduzir as dificuldades dos sistemas anteriores, ao criar condições de micro aeração no reator e promover a interação entre bactérias anaeróbias e aeróbias a partir do controle dos parâmetros como oxigênio dissolvido, carga de amônia, espessura do biofilme, temperatura e concentração de nitrito. São sistemas com baixa demanda de oxigênio, não requerem doador de elétrons, podendo ser realizados também em um único reator.

\section{REFERÊNCIAS}

ABELING, U.; SEYFRIED, C. F.. Anaerobic: aerobic treatment of high strength ammonium wastewater nitrogen removal via nitrite. Water Science and Technology, v.26, p.1007-15, 1992.

AHN, Y. H.. Sustainable nitrogen elimination biotechnologies: A review. Process Biochemistry, v.41, n.8, p.1709-1721, 2006. DOI: https://doi.org/10.1016/j.procbio.2006.03.033

AKUNNA, J. C.; BIZEAU, C.; MOLETTA, R.. Nitrate and nitrite reductions with anaerobic sludge using various carbon sources: Glucose, glycerol, acetic acid, lactic acid and methanol. Water Research, v.27, n.8, p.1303-1312, 1993. DOI: https://doi.org/10.1016/0043-1354(93)90217-6

ARTS, P. A. M.; ROBERTSON, L. A.; KUENEN, J.. GijS Nitrification and denitrification by Thiosphaera pantotropha in aerobic chemostat cultures. FEMS Microbiology Ecology, v.18, n.4, p.305-315, 1995. DOI:

https://doi.org/10.1016/0168-6496(95)00071-2

BAGCHI, S.; BISWAS, R.; NANDY, T.. Alkalinity and dissolved oxygen as controlling parameters for ammonia removal through partial nitritation and Anammox in a single-stage bioreactor. Journal of Industrial Microbiology \& Biotechnology, v.37, p.871-876, 2010.

BUENO, R. D. F.. Nitrificação e Desnitrificação Simultânea em Reator com Biomassa em Suspensão e Fluxo Contínuo de Esgoto. Dissertação (Mestrado em Saúde Pública) Universidade de São Paulo, São Paulo, 2011.

CHARLEY, R. C.; HOOPER, D. G.; MCLEE, A. G.. Nitrification kinetics in activated sludge at various temperatures and dissolved oxygen concentrations. Water Research, v.14, n.10, p.1387-1396, 1980. DOI:

https://doi.org/10.1016/0043-1354(80)90002-0

CHEN, S.; LU, L.; LIN, J.. Biodegradation of tetramethylammonium hydroxide (TMAH) in completely autotrophic nitrogen removal over nitrite (CANON) process. Bioresource Technology, v.210, p.88-93, 2016. DOI: https://doi.org/10.1016/i.biortech.2016.01.127

CHIUMENTI, A.. Complete nitrification-denitrification of swine manure in a full-scale, non-conventional composting system. Waste Management, v.46, p.577-587, 2015. DOI: https://doi.org/10.1016/j.wasman.2015.07.035

CONLEY, D. J.; PAERL, H.W.; HOWARTH, R. W.; BOESCH, D. F.; SEITZINGER, S. P.; HAVENS, K. E.; LANCELOT, C.; LIKENS, G. E.. Controlling eutrophication: nitrogen and phosphorus. Science, v.123, p.1014-1015, 2009. DOI: http://10.1126/science.1167755

DAIMS, H.; LEBEDEVA, E. V.; PJEVAC, P.; HAN, P.; HERBOLD, C.; ALBERTSEN, M.; KIRKEGAARD, R. H.. Complete 
nitrification by Nitrospira bacteria. Nature, v.528, n.7583, p.504-509, 2015. DOI: http://10.1038/nature16461

DANIEL, L. M. C.; POZZI, E.; FORESTI, E.; CHINALIA, F. A. Removal of ammonium via simultaneous nitrificationdenitrification nitrite-shortcut in a single packed-bed batch reactor. Bioresource Technology, v.100, n.3, p.1100-1107, 2009. DOI: https://doi.org/10.1016/j.biortech.2008.08.003

DERKS, Y. M.. Uso da respirometria para aliviar a influência de fatores operacionais e ambientais sobre a ciética de nitrificação. Dissertação (Doutorado) - Universidade Federal de Campina Grande, Campina Grande, 2007.

DIJKMAN, H.; STROUS, M.. Process for ammonium removal from wastewater. PCT/NL99/00446, 1999.

DONG, Z.; SUN, T.. A potential new process for improving nitrogen removal in constructed wetlands-promoting coexistence of partial-nitrification and Anammox. Ecological Engineering, v.31, p.69-78, 2007. DOI: https://doi.org/10.1016/i.ecoleng.2007.04.009

FAN, A. M.; STEINBERG, V. E.. Health implications of nitrate and nitrite in drinking water: an update on methemoglobinemia occurrence and reproductive and developmental toxicity. Regulatory toxicology and pharmacology, v.23, n.1, p.35-43, 1996. DOI: https://doi.org/10.1006/rtph.1996.0006

HANAKI, K.; WANTAWIN, C.; OHGAKI, S.. Nitrification at low level of DO with and without organic loading in a suspended growth reactor. Water Resource, v.24, n.3, p.297-302, 1990. DOI: https://doi.org/10.1016/0043-1354(90)90004-P

HELLINGA, C.; MULDER, J. W.; VAN LOOSDRECHT, M. C. M.; VAN KEMPEN, R.. Full-scale application of theSHARON processfor treatment of rejection water of digested sludge dewatering. Water Science \& Technology, v.43, p.127-134, 2001.

HELLINGA, C.; SCHELLEA, A. A. J. C.; MULDER, J. W.; LOOSDRECHT, M. C. M.; HEIJNEN, J. J.. The SHARON process: an innovative method for nitrogen removal from ammonium-rich wastewater. Water Science Technology, v.9, p.135-142, 1998.

HELMER, C.; KUNST, S.; JURETSCHKO, S.; SCHMID, M. C.; SCHLEIFER, K. H.; WAGNER, M.. Nitrogen loss in a nitrifying biofilm system. Water Science Technology, v.39, n.7, p.13, 1999.

HELMER, C.; TROMM, C.; HIPPEN, A.; ROSENWINKEL, K. H.; SEYFRIED, C. F.; KUNST, S.. Single state nitrogen removal by nitrification and anaerobic ammonium oxidation in biofilm systems. Water Science Technology, v.43, n.20, p.311, 2001.

HIPPEN, A.; ROSENWINKEL, K. H.; BAUMGARTEN G.; SEYFRIED, C. F.. Aerobic deammonification: a new experience in the treatment of wastewaters. Water Science Technology, v.35, p.111-120, 1997.

HU, Y.; ZHAO, X.; ZHAO, Y.. Achieving high-rate autotrophic nitrogen removal via Canon process in a modified single bed tidal flow constructed wetland. Chemical Engineering Journal, v.237, p.329-335, 2014. DOI: https://doi.org/10.1016/j.cej.2013.10.033
JETTEN, M. S. M.; VAN DONGEN, L. G. J. M.; VAN LOOSDRECHT, M. C. M.. The Combined Sharon/Anammox Process. Stowa: Foundation for Applied Water Research. London: IWA Publishing, 2001.

KHIN, T.; ANNACHHATRE, A. P.. Novel microbial nitrogen removal processes. Biotechnology Advances, v.22, n.7, p.519-532, 2004. DOI:

https://doi.org/10.1016/i.biotechadv.2004.04.003

KOCH, G.; EGLI, K.; VAN DER MEER, J. R.; SIEGRIST, H.. Mathematical modeling of autotrophic denitrification in a nitrifying biofilm of a rotating biological contactor. Water Science Technology, v.41, p.191-199, 2000.

KOREN, D. W.; GOULD, W. D.; BÉDARD, P.. Biological removal of ammonia and nitrate from simulated mine and mill effluents. Hydrometallurgy, v.56, n.2, p.127-144, 2000. DOI: https://doi.org/10.1016/S0304-386X(99)00088-2

KUAI, L.; VERSTRAETE, W.. Ammonium removal by the oxygen-limited autotrophic nitrification-denitrification system. Applied and Environmental Microbiology, v.64, n.11, p.4500-4506, 1998.

KUENEN, J. G.. Anammox bacteria: from discovery to application. Nature Reviews: Microbiology, v.6, n 4, p.320326,2008

LAURENI, M.; FALÅS, P.; ROBIN, O.; WICK, A.; WEISSBRODT, D. G.; NIELSEN, J. L.; TERNES, T. A.; MORGENROTH, E.; JOSS A.. Mainstream partial nitritation and anammox: Long-term process stability and effluent quality at low temperatures. Water Research, v.101, p.628-639, 2016. DOI: https://doi.org/10.1016/j.watres.2016.05.005

LI, C.; YANG, J.; WANG, X.; WANG, E.; LI, B.; HE, R.; YUAN, H. Removal of nitrogen by heterotrophic nitrification-aerobic denitrification of a phosphate accumulating bacterium Pseudomonas stutzeri YG-24. Bioresource Technology, v.182, p.18-25, 2015. DOI: https://doi.org/10.1016/j.biortech.2015.01.100

LIANG, Y.; LI, D.; ZHANG, X.; ZENG, H.; YANG, Z.; CUI, S.; ZHANG, J.. Nitrogen removal and microbial characteristics in CANON biofilters fed with different ammonia levels. Bioresource technology, v.171, p.168-174, 2014. DOI: https://doi.org/10.1016/i.biortech.2014.08.072

LIANG, Z.; LIU, J. X.. Control factors of partial nitritation for landfill leachate treatment. Journal of Environmental Science, v.19, p.523-529, 2007.

LOTTI, T.; KLEEREBEZEM, R.; ABELLEIRA-PEREIRA, J. M.; ABBAS, B.; VAN LOOSDRECHT, M. C. M.. Faster through training: The anammox case. Water Research, v.81, p.261268, 2015. DOI: https://doi.org/10.1016/j.watres.2015.06.001

MA, B.; WANG, S.; CAO, S.; MIAO, Y.; JIA, F.; DU, R.; PENG, Y.. Biological nitrogen removal from sewage via anammox: Recent advances. Bioresource Technology, v.200, p.981990, 2016. DOI:

https://doi.org/10.1016/i.biortech.2015.10.074 
METCALF; EDDY. Wastewater engineering: treatment and reuse. Boston: McGraw-Hill, 2003.

MIAO, L.; WANG, S.; CAO, T.; PENG, Y.; ZHANG, M.; LIU, Z. Advanced nitrogen removal from landfill leachate via Anammox system based on Sequencing Biofilm Batch Reactor (SBBR): Effective protection of biofilm. Bioresource Technology, v.220, p.8-16, 2016. DOI: https://doi.org/10.1016/j.biortech.2016.06.131

MULDER, J. W.; VAN KEMPEN, R.. N-removal by SHARON Water Quality International, v.2, p.30-31, 1997.

MÜNCH, E. V.; LANT, P.; KELLER, J.. Simultaneous nitrification and denitrification in bench-scale sequencing batch reactors. Water Research, v.30, n.2, p.277-284, 1996. DOI: https://doi.org/10.1016/0043-1354(95)00174-3

NARKIS, N.; REBHUN, M.; SHEINDORF, C. H.. Denitrification at various carbon to nitrogen ratios. Water Research, v.13, n.1, p.93-98, 1979. DOI: https://doi.org/10.1016/00431354(79)90259-8

OBAJA, D.; MACE, S.; COSTA, J.; SANS, C.; MATA-ALVAREZ, J.. Nitrification, denitrification and biological phosphorus removal in piggery wastewater using a sequencing batch reactor. Bioresource Technology, v.87, n.1, p.103-111, 2003. DOI: https://doi.org/10.1016/S0960-8524(02)00229-8

PARK, Y.; PARK, S.; NGUYEN, V. K.; YU, J.; TORRES, C. I.; RITTMANN, B. E.; LEE, T.. Complete nitrogen removal by simultaneous nitrification and denitrification in flat-panel air-cathode microbial fuel cells treating domestic wastewater. Chemical Engineering Journal, v.316, p.673679, 2017. DOI: https://doi.org/10.1016/j.cej.2017.02.005

QIN, Y.; HAN, B.; CAO, Y.; WANG, T.. Bioresource Technology Impact of substrate concentration on anammox-UBF reactors start-up. Bioresource Technology, v.239, p.422429, 2017. DOI:

https://doi.org/10.1016/j.biortech.2017.04.126

REINO, C.; CARRERA, J.. Low-strength wastewater treatment in an anammox UASB reactor: Effect of the liquid upflow velocity. Chemical Engineering Journal, v.313, p.217-225, 2017. DOI: https://doi.org/10.1016/j.cej.2016.12.051

RIOS-DEL TORO, E. E.; LÓPEZ-LOZANO, N. E.; CERVANTES, F. J.. Up-flow anaerobic sediment trapped (UAST) reactor as a new configuration for the enrichment of anammox bacteria from marine sediments. Bioresource Technology, v.238, p.528-533, 2017. DOI:

https://doi.org/10.1016/j.biortech.2017.04.087

ROBERTSON, L. A.; KUENEN, J. G.. Aerobic denitrification: a controversy revived. Archives of Microbiology, v.139, n.4, p.351-354, 1984.

RYU, J. H.; LEE, H. L.; LEE, Y. P.; KIM, T. S.; KIM, M. K.; ANH, D. T. N.; AHN, D. H.. Simultaneous carbon and nitrogen removal from piggery wastewater using loop configuration microbial fuel cell. Process Biochemistry, v.48, n.7, p.1080-1085, 2013. DOI: https://doi.org/10.1016/j.procbio.2013.05.016

SIEGRIST, H.; REITHAAR, S.; LAIS, P.. Nitrogen loss in a nitrifying rotating contactor treating ammonium rich leachate without organic carbon. Water Science

Technology, v.37, p.589, 1998.

SILVA FILHO, H. A.. Nitrificação em Sistemas de Lodo Ativado. 2009

SLIEKERS, O. A.; DERWORT, N.; CAMPOS-GOMEZ, J. L.; STROUS, M.; KUENEN, J. G.; JETTEN, M. S. M.. Completely autotrophic nitrogen removal over nitrite in a single reactor Water Research, v.36, p.2475-2482, 2002. DOI: https://doi.org/10.1016/S0043-1354(01)00476-6

SLIEKERS, O. A.; THIRD, K.; ABMA, W.; KUENEN, J. G.; JETTEN, M. S. M.. CANON and Anammox in a gas-lift reactor. FEMS Microbiology Letters, v.218, p.339-344, 2003. DOI: https://doi.org/10.1016/S0378-1097(02)01177-1

SMITH, V. H.; TILMAN, G. D.; NEKOLA, J. C.. Eutrophication: impacts of excess nutrient inputs on freshwater, marine, and terrestrial ecosystems. Environmental pollution, v.100, n.1, p.179-196, 1999. DOI: https://doi.org/10.1016/S02697491(99)00091-3

SPAGNI, A.; MARSILI-LIBELLI, S.; LAVAGNOLO, M. C.. Optimisation of sanitary landfill leachate treatment in a sequencing batch reactor. Water Science Technology, v.58, p.337-343, 2008.

SPAGNI, A.; MARSILI-LIBELLI, S.. Nitrogen removal via nitrite in a sequencing batch reactor treating sanitary landfill leachate. Bioresource Technology, v.100, p.609-614, 2009. DOI: https://doi.org/10.1016/j.biortech.2008.06.064

SRI SHALINI, S.; JOSEPH, K.. Nitrogen management in landfill leachate: Application of SHARON, ANAMMOX and combined SHARON-ANAMMOX process. Waste Management, v.32, p.2385-2400, 2012. DOI: https://doi.org/10.1016/i.wasman.2012.06.006

STROUS, M.; FUERST, J. A.; KRAMER, E. H.; LOGEMANN, S. MUYZER, G.; VAN DE PAS-SCHOONEN, K. T.; WEBB, R.; KUENEN, J. G.; JETTEN, M. S.. Missing lithotroph identified as new planctomycete. Nature, v.400, n.6743, p.446-449, 1999.

STROUS, M.; HEIJNEN, J. J.; KUENEN, J. G.; JETTEN, M. S. M.. The sequencing batch reactor as a powerful tool for the study of slowly growing anaerobic ammonium-oxidizing microorganisms. Applied Microbiology and Biotechnology, v.50, n.5, p.589-596, 1998.

SUN, Z.; LV, Y.; LIU, Y.; REN, R.. Removal of nitrogen by heterotrophic nitrification-aerobic denitrification of a novel metal resistant bacterium Cupriavidus sp. S1. Bioresource Technology, v.220, p.142-150, 2016. DOI: https://doi.org/10.1016/j.biortech.2016.07.110

SURAMPALLI, R. Y.; TYAGI, R. D.; SCHEIBLE, O. K.; HEIDMAN, J. A.. Nitrification, denitrification and phosphorus removal in sequential batch reactors. Bioresource Technology, v.61, n.2, p.151-157, 1997. Dol: https://doi.org/10.1016/S09608524(97)00034-5

SUTO, R.; ISHIMOTO, C.; CHIKYU, M.; AIHARA, Y. MATSUMOTO, T.; UENISHI, H.; YASUDA, T.; FUKUMOTO, Y.; WAKI, M.. Anammox biofilm in activated sludge swine wastewater treatment plants. Chemosphere, v.167, p.300- 
307, 2017. DOI:

https://doi.org/10.1016/i.chemosphere.2016.09.121

TANG, C.; ZHENG, P.; WANG, C.; MAHMOOD, Q.; ZHANG, J.; CHEN, X.; ZHANG, L.; CHEN, J.. Performance of high-loaded ANAMMOX UASB reactors containing granular sludge. Water Research, v.45, n.1, p.135-144, 2011. DOI: https://doi.org/10.1016/j.watres.2010.08.018

TIMBERLAKE, D. L.; STRAND, S. E.; WILLIAMSON, K. J.. Combined aerobic heterotrophic oxidation, nitrification and denitrification in a permeable-support biofilm. Water Research, v.22, n.12, p.1513-1517, 1988. DOI: https://doi.org/10.1016/0043-1354(88)90163-7

TOMASZEWSKI, M.; CEMA, G.; ZIEMBIŃSKA-BUCZYŃSKA, A.. Influence of temperature and $\mathrm{pH}$ on the anammox process: A review and meta-analysis. Chemosphere, v.182, p.203214, 2017. DOI:

https://doi.org/10.1016/j.chemosphere.2017.05.003

VAN DE GRAAF, A. A.; MULDER, A.; DE BRUIJN, P.; JETTEN, M. S. M.; ROBERTSON, L. A.; KUENEN, J. G.. Anaerobic oxidation of ammoinium is a biologically mediated process. Applied and Environmental Microbiology, v.61, n.4, p.12461251, 1995.

VAN DONGEN, U.; JETTEN, M. S. M.; VAN LOOSDRECHT, M. C. M.. The SHARON-ANAMMOX process for treatment of ammonium rich wastewater. Water Science Technolology, n.44, p.153-160, 2001.

VAN HULLE, S. W. H.; VANDEWEYER, H. J. P.; MEESSCHAERT, BOUDEWIKN, D.; VANROLLEGHEM, P. A.; DEJANS, P.; DUMOULIN, A. N. N.. Engineering aspects and practical application of autotrophic nitrogen removal from nitrogen rich streams. Chemical Engineering Journal, v.162, p.1-20, 2010. DOI: https://doi.org/10.1016/i.cej.2010.05.037

VAN KEMPEN, R.; MULDER, J. W.; UIJTERLINDE, C. A.; LOOSDRECHT, M. C. M.. Overview: full scale experience of the SHARON ${ }^{\circledR}$ process for treatment of rejection water of digested sludge dewatering. Water Science and Technology, v.44, n.1, p.145-152, 2001.

VERSTRAETE, W.; PHILIPS, S.. Nitrification-denitrification processes and technologies in new contexts. Environmental Pollution, v.102, p.717-72, 1998. DOI:

http://doi.org/10.1016/S0269-7491(98)80104-8
VIRDIS, B.; RABAEY, K.; ROZENDAL, R. A.; YUAN, Z.; KELLER, J.. Simultaneous nitrification, denitrification and carbon removal in microbial fuel cells. Water Research, v.44, n.9, p.2970-2980, 2010. DOI: https://doi.org/10.1016/j.watres.2010.02.022

VITOUSEK, P. M.; ABER, J. D.; HOWARTH, R. W.; LIKENS, G. E.; MATSON, P. A.; SCHINDLER, D. W.; SCHLESINGER, W. H.; TILMAN, D. G.. Human alteration of the global nitrogen cycle: sources and consequences. Ecological applications, v.7, n.3, p.737-750, 1997.

WEI, S. H.; QING, Y.; GUORI, D.; HONGXUN, H.; SHUJUN, Z.; YING, Y. Y.; YONG, P. Z.. Achieving the nitrite pathway using FA inhibition and process control in UASB-SBR system removing nitrogen from landfill leachate. Science China Chemical, v.53, p.1210-1216, 2010.

WEN, X.; GONG, B.; ZHOU, J.; HE, Q.; QING, X.. Efficient simultaneous partial nitrification, anammox and denitrification (SNAD) system equipped with a real-time dissolved oxygen (DO) intelligent control system and microbial community shifts of different substrate concentrations. Water Research, v.119, p.201-211, 2017. DOI: https://doi.org/10.1016/j.watres.2017.04.052

WINOGRADSKY, S.. Recherches sur les organismes de la nitrification. Ann. Inst. Pasteur, Massoon, v.4, p.213-231, 1890.

YOO, H.; AHN, K. H.; LEE, H. J.; LEE, K. H.; KWAK, Y. J.; SONG, K. G.. Nitrogen removal from synthetic wastewater by simultaneous nitrification and denitrification (SND) via nitrite in an intermittently-aerated reactor. Water Research, v.33, n.1, p.145-154, 1999. DOI: https://doi.org/10.1016/S00431354(98)00159-6

ZHANG, L.; NARITA, Y.; GAO, L.; ALI, M.; OSHIKI, M.; OKABE, S.. Maximum specific growth rate of anammox bacteria revisited. Water Research, v.116, p.296-303, 2017. DOI: https://doi.org/10.1016/j.watres.2017.03.027

ZHANG, Z.; LIU, S.. Hot topics and application trends of the anammox biotechnology: a review by bibliometric analysis. SpringerPlus, v.3, n.1, p.220-227, 2014.

ZOPPAS, F. M.; BERNARDES, A. M.; MENEGUZZI, A.. Parâmetros operacionais na remoção biológica de nitrogênio de águas por nitrificação e desnitrificação simultânea.

Engenharia Sanitária e Ambiental, v.21, n.1, p. 29-42, 2016.

A CBPC - Companhia Brasileira de Produção Científica (CNPJ: 11.221.422/0001-03) detém os direitos materiais desta publicação. Os direitos referem-se à publicação do trabalho em qualquer parte do mundo, incluindo os direitos às renovações, expansões e disseminações da contribuição, bem como outros direitos subsidiários. Todos os trabalhos publicados eletronicamente poderão posteriormente ser publicados em coletâneas impressas sob coordenação da Sustenere Publishing, da Companhia Brasileira de Produção Científica e seus parceiros autorizados. Os (as) autores (as) preservam os direitos autorais, mas não têm permissão para a publicação da contribuição em outro meio, impresso ou digital, em português ou em tradução. 